\title{
On Calculation of the Ground Transient Resistance of Overhead Lines
}

\author{
Sergey L. Loyka
}

\begin{abstract}
A constraint on the calculation of the ground transient resistance of overhead lines for small values of time is pointed out. The method for overcoming this constraint is proposed. The source of the ground transient resistance singularity is the low-frequency approximation of the ground impedance. It is shown that the ground transient resistance is not singular for $t \rightarrow 0$ if it is calculated without the low-frequency approximation.
\end{abstract}

Index Terms-Ground impedance, lighting, overhead lines.

\section{INTRODUCTION}

$\mathbf{T}$ HE ground impedance has considerable effect on lightning-induced voltages on overhead lines. Many approximate equations for the field-to-transmission linecoupling calculation taking into account this impedance have been proposed [1]-[5]. In the present paper, we consider some constraints on the ground impedance calculation in the time-domain and propose a way to overcome these constraints.

The expression for the per-unit-length ground impedance in the frequency domain for a single wire located at height $h$ above ground can be written as follows [3], [4]:

$$
Z_{g}^{\prime}(\omega)=\frac{j \omega \mu_{0}}{\pi} \int_{0}^{\infty} \frac{e^{-2 h x}}{\sqrt{x^{2}+\gamma_{g}^{2}}+x} d x
$$

where $\gamma_{g}$ is the propagation constant in the ground

$$
\gamma_{g}(\omega)=\sqrt{j \omega \mu_{0} \sigma_{g}-\omega^{2} \mu_{0} \epsilon_{g}}
$$

$\sigma_{g}$ is the ground conductivity, $\epsilon_{g}$ is the ground permittivity, and $\mu_{0}$ is the permeability of the free-space.

The electric field component due to the ground impedance is

$$
E_{g}(\omega)=Z_{g}^{\prime}(\omega) I(\omega)
$$

where $I(\omega)$ is the line current. In the time domain, this equation takes the following form:

$$
e_{g}(t)=z_{g}(t) * i(t)
$$

where $z_{g}(t)$ denotes the inverse Fourier transform of $Z_{g}^{\prime}(\omega)$ and " $*$ " denotes the convolution. The so-called ground transient resistance, which is given by the inverse Fourier transform

$$
\xi(t)=\boldsymbol{F}^{-1}\left(\frac{Z_{g}^{\prime}}{j \omega}\right)
$$

Manuscript received May 21, 1998; revised March 22, 1999.

The author is with the Belorussian State University of Informatics and Radioelectronics, Minsk, 220027 Belarus.

Publisher Item Identifier S 0018-9375(99)06719-8. can also be used for $e_{g}(t)$ calculation

$$
e_{g}(t)=\xi(t) * \frac{d i(t)}{d t}
$$

The term "ground transient resistance" is used for $\xi(t)$ because it is a time-domain function and its dimension is Ohms/meter [2], [3], [5]. For a low-frequency approximation, when

$$
\omega \ll \omega_{\max }=\frac{\sigma_{g}}{\epsilon_{g}}
$$

and only the first term in (2) is essential, $\xi(t)$ can be calculated by Timotin's formula [1]-[4]

$$
\begin{aligned}
& \xi(t)= \frac{\mu_{0}}{\pi \tau_{g}}\left[\frac{1}{2 \sqrt{\pi}} \sqrt{\frac{\tau_{g}}{t}}+\frac{1}{4} \exp \left(\frac{\tau_{g}}{t}\right)\right. \\
&\left.\cdot \operatorname{erfc}\left(\sqrt{\frac{\tau_{g}}{t}}\right)-\frac{1}{4}\right]
\end{aligned}
$$

where $\tau_{g}=h^{2} \mu_{0} \sigma_{g}$, and $\operatorname{erfc}(*)$ is the complementary error function

$$
\operatorname{erfc}(x)=\frac{2}{\sqrt{\pi}} \int_{x}^{\infty} e^{-t^{2}} d t
$$

As it is pointed out in [4] and [5], (5) has singular behavior at $t=0$ and

$$
\xi(t) \approx \frac{\mu_{0}}{2 \pi} \frac{1}{\sqrt{\pi \tau_{g} t}} \text { for } t \rightarrow 0 .
$$

However, the following question arises. "Is this singularity a real one or is it a consequence of the low frequency approximation?" As will be shown, the source of this singularity is the low-frequency approximation and it will not exist if $\xi(t)$ is calculated without the low-frequency assumption.

\section{Constraint On THE Ground Transient Resistance Calculation}

Since (8) has been obtained in the low-frequency approximation, it is not valid for small values of $t$. We will explain it in the following example [6, p. 189]. Let's consider the inverse Fourier transform of function $Y(\omega)$

$$
y(t)=\frac{1}{2 \pi} \int_{-\infty}^{\infty} Y(w) e^{j \omega t} d \omega .
$$

If $Y(\omega)$ is a constant, then $y(t)=0$ for all $t \neq 0$ [due to the oscillating factor $e^{j \omega t}$ in the integrand in (11)]. If $Y(\omega)$ is not a constant but it is varied slowly enough in comparison to $e^{j \omega t}$, then the infinite integration limits can be reduced to the finite 
ones since the integration subinterval for which $|\omega| t \gg 1$ does not give a substantial contribution to the total integral due to the fast-oscillating factor $e^{j \omega t}$; the subinterval for which $|\omega| t<1$ gives a substantial contribution since the factor $e^{j \omega t}$ does not oscillate in this subinterval. Further, we will carry out simple order-of-magnitude calculations. Taking into account the considerations given above, (11) can be reduced to

$$
y(t) \approx \frac{1}{2 \pi} \int_{-\omega_{\max }}^{\omega_{\max }} Y(w) e^{j \omega t} d \omega .
$$

where

$$
\omega_{\max } \approx \frac{1}{t}
$$

Thus, the spectrum of $y(t)$ must be known up to $\omega_{\max } \approx$ $1 / t_{0}$ in order to calculate $y(t)$ at the point $t_{0}$. In a similar manner, if we know the function $Y(\omega)$ up to $\omega_{\max }$, then we can calculate $y(t)$ for $t \geq 1 / \omega_{\max }$. If $y(t)$ must be calculated for smaller values of $t, Y(\omega)$ has to be known for larger values of $\omega$.

Let us now turn our attention to $\xi(t)$. Since $Z_{g}(\omega)$ in the low-frequency approximation is known up to $\omega_{\max }=\sigma_{g} / \epsilon_{g}$ [see (7)], we can correctly calculate $\xi(t)$ using (8) for

$$
t \geq t_{\min }=\frac{\epsilon_{g}}{\sigma_{g}} .
$$

The calculation of $\xi(t)$ using (8) for $t<t_{\min }$ is not correct.

\section{Calculation of the Ground Transient RESISTANCE FOR SMALL VAlUES OF TIME}

In this case, $\xi(t)$ can be calculated with order-of-magnitude accuracy as follows. Let us transform (5) to

$$
\xi(t)=\frac{1}{2 \pi} \int_{0}^{\infty} \frac{Z_{g}^{\prime}}{j \omega} e^{j \omega t} d \omega+\frac{1}{2 \pi} \int_{-\infty}^{0} \frac{Z_{g}^{\prime}}{j \omega} e^{j \omega t} d \omega .
$$

Taking into account that $\left|Z_{g}^{\prime}(-\omega)\right|=\left|Z_{g}^{\prime}(\omega)\right|$ and $\varphi_{g}(-\omega)=-\varphi_{g}(\omega)$ [it can be easily verified by means of (1) and (2)], where $\varphi_{g}(\omega)$ is the phase of $Z^{\prime} g(\omega)$, after some manipulations we obtain

$$
\xi(t)=\frac{1}{\pi} \int_{0}^{\infty} \frac{\left|Z_{g}\right|}{\omega} \sin \left(\omega t+\varphi_{g}\right) d \omega .
$$

Taking into account the considerations given in the previous section, this equation can be reduced to

$$
\xi(t) \approx \frac{1}{\pi} \int_{0}^{t^{-1}} \frac{\left|Z_{g}\right|}{\omega} \sin \left(\omega t+\varphi_{g}\right) d \omega .
$$

Let's make the following approximation: since $\omega \cdot t \leq 1, \omega \cdot t$ in (17) can be neglected with order-of-magnitude accuracy. In fact, it means that we present $\sin \left(\omega \cdot t+\varphi_{g}\right)$ as $\cos (\omega$. t) $\sin \left(\varphi_{g}\right)+\sin (\omega \cdot t) \cos \left(\varphi_{g}\right)$ and make the approximations $\cos (\omega \cdot t) \approx 1$ and $\sin (\omega \cdot t) \approx 0$ for small values of $\omega \cdot t$ $(\omega \cdot t \leq 1)$. Further, we divide the total integration interval $\left[0, t^{-1}\right]$ into two subintervals $\left[0, t_{\mathrm{min}}^{-1}\right]$ and $\left[t_{\mathrm{min}}^{-1}, t^{-1}\right]$, where $t_{\min }$ is given by (14). Thus, we obtain

$$
\begin{aligned}
\xi(t) \approx & \frac{1}{\pi} \int_{0}^{t_{\max }^{-1}} \frac{\left|Z_{g}\right|}{\omega} \sin \left(\varphi_{g}\right) d \omega \\
& +\frac{1}{\pi} \int_{t_{\min }^{-1}}^{t^{-1}} \frac{\left|Z_{g}\right|}{\omega} \sin \left(\varphi_{g}\right) d \omega .
\end{aligned}
$$

But the first integral in (18) can be calculated by (8) since this equation is valid for the interval $\left[0, t_{\min }^{-1}\right]$. The second integral in (18) we estimate as follows. It is known [3] that for $\omega \rightarrow \infty$

$$
Z_{g}^{\prime}(\omega) \rightarrow Z_{\lim }^{\prime}=\frac{1}{2 \pi h} \sqrt{\frac{\mu_{0}}{\epsilon_{g}}} .
$$

Consequently, $\varphi_{g} \rightarrow 0$. We can use the approximation $Z_{g}^{\prime}(\omega)=Z_{\lim }^{\prime}$ for $\omega>\omega_{\mathrm{lim}}$, where the limiting frequency $\omega_{\text {lim }}$ can be estimated as follows. The upper integration limit in (1) can be reduced to $x_{\max } \approx 10 / h$ because of the exponential factor in the integrand. $Z_{g}^{\prime}(\omega)$ does not depend on $\omega$ if $\gamma_{g} \gg x_{\max }$. Thus, ( $\omega_{\lim }$ can be estimated from the following equation:

$$
\left|\gamma_{g}(\omega)\right|=x_{\max }
$$

For sufficiently high frequencies, $\left|\gamma_{g}(\omega)\right| \approx \omega \sqrt{\mu_{0} \epsilon_{g}}$ and, consequently

$$
\omega_{\lim } \approx \frac{x_{\max }}{\sqrt{\epsilon_{g} \mu_{0}}} \approx \frac{10}{h \sqrt{\epsilon_{g} \mu_{0}}} .
$$

In a general case (for large values of $\sigma_{g}$ ), one must take into consideration both terms in (2); then (21) should be replaced by

$$
\omega_{\lim } \approx \max \left[\frac{10}{h \sqrt{\epsilon_{g} \mu_{0}}}, 10 \omega_{\max }\right] .
$$

where $\omega_{\max }$ is given by (7). Thus, starting from $\omega_{\text {lim }}$ we can use (19) as a good approximation for $Z_{g}(\omega)$. Then, using (18) for sufficiently small values of $t$ (namely, for $t<1 / \omega_{\text {lim }}$ ), we obtain the following estimation for $\xi(t)$ :

$$
\xi(t) \approx \xi_{\max } \approx \xi_{T}\left(t_{\min }\right)+\frac{Z_{\lim }}{\pi} \ln \left(t_{\min } \omega_{\lim }\right)
$$

where $\xi_{T}(t)$ is calculated by (8). While obtaining this equation, we reduced the upper limit in the second integral in (18) from $t^{-1}$ down to $\omega_{\mathrm{lim}}$ since $\varphi_{g} \approx 0$ for $\omega>\omega_{\mathrm{lim}}$ and made the following approximations: $\sin \varphi_{g} \approx 1$ and $\left|Z_{g}^{\prime}\right| \approx Z_{\lim }^{\prime}$ for the interval $\left[t_{\min }^{-1}, \omega_{\lim }\right]$.

Equation (23) clearly shows that $\xi(t) \rightarrow$ const for $t \rightarrow 0$ rather than is divergent as in (10). Numerical calculations by means of (1), (2), and (5) prove (23) and, thus, all the approximations we made above (within order-of-magnitude accuracy). Fig. 1 shows the transient resistance computed by (1), (2), and (5), by the Timotin's formula (8) and the limiting formula (23). It's evident that the transient resistance approaches a constant when the time tends to zero. The 


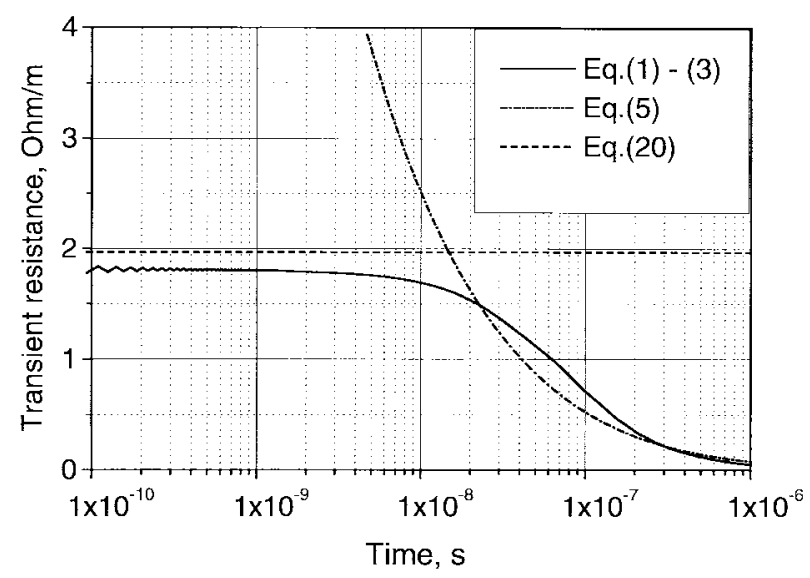

Fig. 1. Comparison of various transient resistance formulas: $h=10$, $\epsilon_{g} / \epsilon_{0}=10, \sigma_{g}=10^{-3}$.

limiting value (23) gives quite a good approximation to the value obtained by (1), (2), and (5).

The above equations give us possibility to calculate both the approximate value of $\lim _{t \rightarrow 0} \xi(t)$ and the limiting time $\left(1 / \omega_{\mathrm{lim}}\right)$ starting from which $\xi(t)$ is nearly constant. The exact value of $\lim _{t \rightarrow 0} \xi(t)$ can also be calculated by means of the initial value theorem for the Laplace transform [7]

$$
\lim _{t \rightarrow 0} x(t)=\lim _{s \rightarrow \infty} s X(s)
$$

where $x(t)$ is a time-domain function and $X(s)$ is its Laplace transform. Using (1), (2), (19), and (24), we obtain

$$
\lim _{t \rightarrow 0} \xi(t)=Z_{\lim }=\frac{1}{2 \pi h} \sqrt{\frac{\mu_{0}}{\epsilon_{g}}} .
$$

Using this equation and the above considerations, we can recommend the following equation for $\xi(t)$ for any value of $t$ :

$$
\xi(t)=\min \left[\xi_{T}(t), Z_{\lim }\right] .
$$

In order to understand why $\xi_{T}(t)$ is divergent for $t \rightarrow 0$, it is necessary to calculate $\lim _{\omega \rightarrow \infty} Z_{g}^{\prime}(\omega)$ for the low frequency approximation, when $\gamma_{g}^{2}(\omega) \approx j \omega \mu_{0} \tau_{g}$ [it is this approximation that was used in (8)]. Using (1), one obtains in this case

$$
\lim _{\omega \rightarrow \infty} Z_{g}(\omega)=\frac{1}{2 \pi h} \sqrt{\frac{j \omega \mu_{0}}{\sigma_{g}}} .
$$

It is evident that $\left|Z_{g}^{\prime}(\omega)\right| \sim \sqrt{\omega}$ and $\varphi_{g}(\omega) \sim \pi / 4$ for $\omega \rightarrow \infty$. Referring to (18), we obtain for $\xi(t)$ the divergence of the kind $1 / \sqrt{t}$ for $t \rightarrow 0$. Thus, the cause of this divergence lies in the fact that the low-frequency approximation gives incorrect behavior of the amplitude and phase of $Z_{g}^{\prime}(\omega)$ for $\omega \rightarrow \infty$.

\section{CONCLUSION}

Thus, Timotin's formula for the ground transient resistance gives a significant error for small values of time. The minimum value of time for which this formula is still valid depends on the ground parameters. The simple order-of-magnitude method of the ground transient resistance calculation for small values of time is proposed above.

It should be noted that the ground transient resistance can be calculated in an efficient way by (1), (2), and (5) using inverse fast Fourier transform for (5) with a large number of samples. The only constraint from the viewpoint of computational efficiency is the computing of integral (1) for a large number of frequencies. This constraint can be overcome by computing integral (1) for a small number of frequencies and using an interpolation for the other frequencies. It's also possible to improve the computational efficiency by using the fact that the ground impedance is nearly constant for rather high frequencies [see (19)]. The computation time for this method on a Pentium PC for FFT with $2^{19}$ points is only several minutes. An alternative way is to use the Sunde's formula for the ground impedance, which is a good approximation to (1) and does not require any integration [3].

\section{ACKNOWLEDGMENT}

The author would like to thank Prof. V. A. Lipnitzkii for many useful discussions on the asymptotic methods and the reviewers whose comments helped to improve the paper.

\section{REFERENCES}

[1] A. Timotin, "Longitudinal transient parameters of a unifilar line with ground return," Rev. Roum. Sci. Tech., Electrotech. Energie, vol. 12, no. 4, pp. 523-535, 1967.

[2] E. S. M. Mok and G. I. Costashe, "Skin-effect consideration on transient response of a transmission line excited by an electromagnetic pulse," IEEE Trans. Electromagn. Compat., vol. 34, pp. 320-329, Aug. 1992.

[3] F. Rachidi, C. A. Nucci, M. Ianoz, and C. Mazzetti, "Influence of a lossy ground on lightning-induced voltages on overhead lines," IEEE Trans. Electromagn. Compat., vol. 38, pp. 250-264, Aug. 1996.

[4] F. Tesche, M. Ianoz, and T. Karlsson, EMC Analysis Methods and Computational Models. New York: Wiley, 1997.

[5] D. Orzan, "Time-domain low frequency approximation for the offdiagonal terms of the ground impedance matrix," IEEE Trans. Electromagn. Compat., vol. 39, p. 64, Feb. 1997.

[6] L. D. Landau and E. M. Lifshits, Field Theory. Moscow, Russia: Nauka, 1973.

[7] G. A. Korn and T. M. Korn, Mathematical Handbook. New York: McGraw-Hill, 1968.

Sergey L. Loyka, photograph and biography not available at the time of publication. 